\title{
Marionina istriae n. sp., ein mariner Enchytraeide (Oligochaeta) aus dem mediterranen Hygropsammal
}

\author{
O. GIERE \\ Zoologisches Institut und Zoologisches Museum der Universität Hamburg; \\ Hamburg 13, Bundesrepublik Deutschland
}

\begin{abstract}
Marionina istriae n. sp., a marine enchytraeid (Oligochaeta) from the Mediterranean hygropsammal. A new enchytraeid worm, Marionina istriae n. sp. (Oligochaeta), is described from the marine supralittoral of an island near Rovinj (Yugoslavian coast of the Adriatic Sea), The position of the new species within the genus Marionina is discussed. A short diagnosis of the new species is given by a diagrammatic illustration of some taxonomically important anatomical features. The discussion includes some considerations on the relevance of brain size and arrangement of blood vessels for enchytraeid systematics.
\end{abstract}

\section{EINLEITUNG}

Während eines marin-zoologischen Ferienkurses am Ruder-Bošković-Institut in Rovinj (Istrien, Jugoslawien) wurden im Hygropsammal Enchytraeiden gefunden, die nicht eindeutig zuzuordnen waren. Die originalen Sedimentproben mit den lebenden Oligochaeten wurden daraufhin in Hamburg näher bearbeitet. Die vorliegende Arbeit befaßt sich mit den Ergebnissen dieser taxonomischen und ökologischen Untersuchungen.

\section{MATERIAL UND METHODE}

Die vorliegende Untersuchung umfaßt die mikroskopische Totalanalyse von ca. 20 vollreifen lebenden Tieren, etwa gleich vielen lebenden subadulten und von 13 fixierten Individuen. Die Fixierung und Färbung wurde mit der nach bisherigen Erfahrungen besten Methode für Enchytraeiden (auch für Totaluntersuchungen) durchgeführt, die REISINGER (1925) beschrieb. Für die angegebenen Maßzahlen ist von Bedeutung, daß sie auf Lebendausmessungen beruhen, kombiniert mit der Analyse fixierten Materials.

\section{ERGEBNISSE}

Die Artbeschreibung von Marionina istriae n. sp. konzentriert sich im wesentlichen auf die stichwortartige Darstellung der diagnostisch wichtigen Kriterien; sie bean- 
sprucht daher keine Vollständigkeit in der Erörterung allgemeiner anatomischer Merkmale.

$\mathrm{H} \mathrm{a} \mathrm{b} \mathrm{it} \mathrm{u} \mathrm{s} \mathrm{:} \mathrm{Länge} \mathrm{ca.} 7$ bis $10 \mathrm{~mm}$, oft $8 \mathrm{~mm}$. Breite in Körpermitte ca. 0,2 mm. Segmentzahl reifer Tiere zwischen 38 und 43 schwankend, häufig 41 . Farbe weiß, Chloragocyten im Durchlicht grünlich. In jedem Segment ca. 7 Reihen von Hautdrüsen. Clitellum in den Segmenten 12 bis $1 / 213$ gelegen, wenig hervortretend und daher nicht besonders auffällig. Prostomium und Pygidium ohne Besonderheiten für das Genus. After terminal.

B orste n: Mit ca. $65 \mu \mathrm{m}$ Länge recht groß relativ zum Körperdurchmesser. Borstenlänge ante- und postclitellar etwa gleich. Borstenmuskulatur stark entwickelt. Borsten typisch marionin: fast gerade, mit inneren "Haken“, ektal spitz endend. Borsten im Bündel etwa gleich lang. Konstant 2 Borsten pro Bündel. Im jedem Körpersegment 2 Paar Borstenbündel, Borstenformel somit $2: 2-2: 2$. Segment 1 wie üblich ohne Borsten, Segment 2 voll beborstet, Segment 12 ( $\delta$ Geschlechtsöffnung) ohne Ventralborsten, letztes echtes Segment mit Borsten.

Ge h ir $\mathrm{n}$ : In Segmenten 1 und 2 gelegen, Vorderrand leicht konkav, Hinterrand gewöhnlich gerade (Abb. 1a), gelegentlich auch schwach konvex. Ganz vereinzelt erschien in einigen Bewegungsphasen der Hirn-Hinterrand jedoch auch deutlich ausgebuchtet (Abb. 1b, vgl. p. 367).

Verda u n g trakt: Darm gerade gestreckt, Schlundkopf wie üblich mit starker Muskulatur. Peptonephridien fehlen. Oesophagus recht englumig, sich allmählich zum Mitteldarm erweiternd. Ohne Oesophagialdivertikel. Septaldrüsen an Dissepimenten $4 / 5,5 / 6$ und $6 / 7$ entwickelt. Die dorsalen Teile der beiden vorderen Paare jeweils verschmolzen, die letzten Drüsen nur lateral entwickelt (Abb. 3). Ausfuhrgänge zum Pharynx hin nicht auffällig stark erkennbar. Oesophagus ab Segment 6 mit Chloragocyten dicht besetzt.

Coelo m: In der Coelomflüssigkeit zahlreiche scheibenförmige Lymphocyten von ovalem bis unregelmäßig birnförmigem Umriß. Länge ca. $34 \mu \mathrm{m}$, Breite ca. $13 \mu \mathrm{m}$; mit großem zentralen Kern im körnig granulierten Plasma.

B 1 u t g e f ä $B$ s y s t e m : Ursprung des Dorsalgefäßes in Mitte des 13. Segments, also im Bereich des Clitellums. Aufzweigung in die beiden vordersten Lateralschlingen in Segment 3 (Abb. 1c). Lateralschlingen erstrecken sich latero-rostrad bis in Segment 2 hinein, um sich dann ventral in ca. ${ }^{4 / 5}$ wieder zum Ventralgefäß zu vereinen. Dicht hinter der vordersten Aufzweigung geht dorsal ein weiteres Paar von Lateralgefäßen in Segment 4 ab. Das Blut ist farblos.

Nephridien: (Abb. 2a). Recht groß und massiv; Anteseptale : Postseptale $=$ ca. $1: 3$. Nephridialtrichter weit, Offnung schräg ansetzend. Im relativ großen anteseptalen Teil mehrere Windungen des Exkretionskanals deutlich. Dieser im Postseptale vielfach gewunden. Am Ubergang Ante- zu Postseptale keine ausgeprägte Einschnürung. Ausführgang kurz und gerade, entspringt am Hinterende des Postseptale.

Männliches Genitalsystem und Anhangsorgane: Testes in Segment 11, kompakt, mit unregelmäßigem und unscharf abgegrenztem Hinterrand. Keine Seminalvesikel vorhanden. Klumpen von Spermatocyten verschiedenen Reifegrades daher frei im Coelom der Segmente 11 und 12. Samentrichter (Abb. 1e und 2b) kompakt, Länge mehr als die halbe Breite des Tieres, ungefähr $110 \mu \mathrm{m}$. Breite etwa $1 / 5$ 


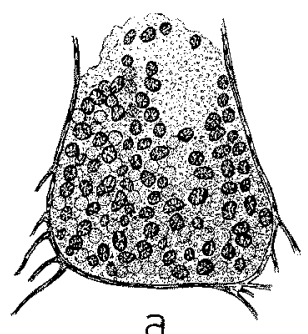

a

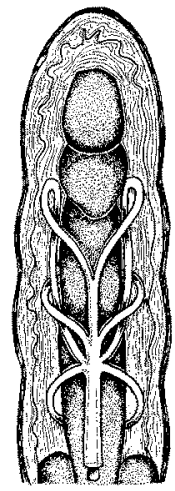

C

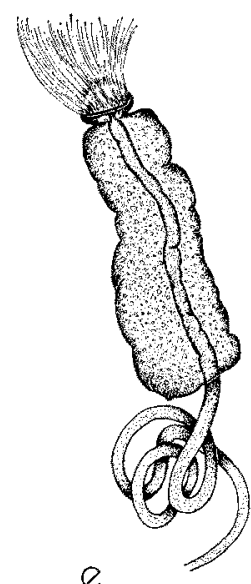

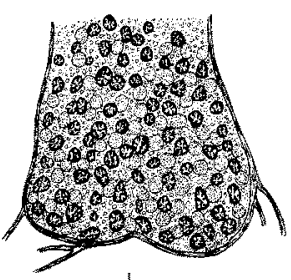

b

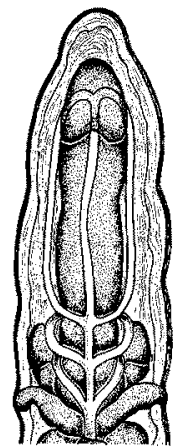

d

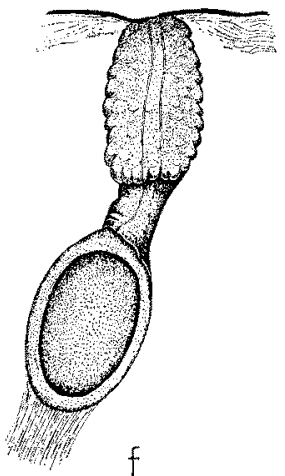

Abb. 1: Marionina istriae n. sp. soweit nicht anders erwähnt. Alle Zeichnungen nach Lebendaufnahmen; $a$ Gehirn mit geradem Hinterrand (Normalform); $b$ Gehirn mit eingebuchtetem Hinterrand; $c$ Aufzweigung des dorsalen Blutgefäßes in Segment 3 bis 4; $d$ Lumbricillus lineatus. Aufzweigung des dorsalen Blutgefäßes in Segment 1; $e$ Samentrichter mit Samenleiter in Segment 12; $f$ Spermatheka in normaler Form: Ampulle rundlich-oval. 


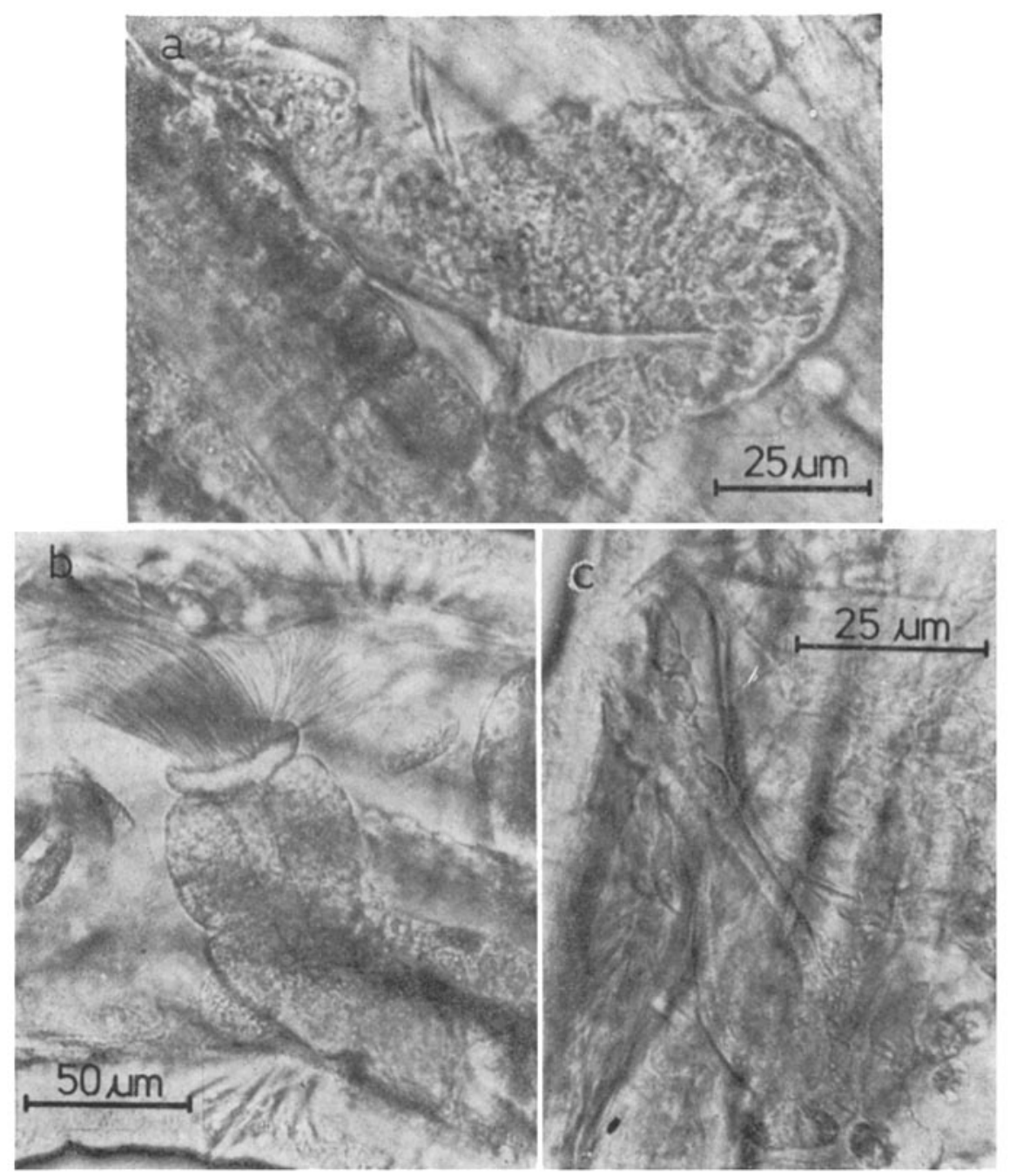

Abb. 2: Marionina istriae n. sp. a Nephridium von ventro-lateral; $b$ Samentrichter mit Spermien; $c$ Spermatheka, leicht gestreckt. Drüsen am Ausführgang deutlich. (Lebendaufnahmen, Mikroblitz)

der Gesamtbreite, etwa $40 \mu \mathrm{m}$. Je nach Streckungsgrad Länge zu Breite des Samentrichters ca. $3: 1$ (bis $4: 1$ ). Seitenränder des Samentrichters unregelmäßig schwach gelappt. Samentrichter besitzt bei vollreifen Tieren einen deutlich abgesetzten Kragen von ca. $3 / 4$ des Gesamtdurchmessers des Trichters. Bei subadulten Individuen Kragen schwächer entwidkelt und ohne gewulsteten Rand. Doch auch bei diesen Tieren Trichter schon früh besetzt mit einem langen Schopf von Spermien. Der Samenleiter ist im drüsigen Trichterbereich leicht erweitert und gut erkennbar. Hinterer Teil des Samenlei- 
ters ist relativ lang und verläut unregelmäßig in 3 bis 4 Spiralwindungen im Segment 12 (Abb. 1e). Penialbulbus kompakt, sich wenig über die Epidermis-Oberfläche heraushebend. Penialdrüsen recht stark entwickelt, deutlich in das Coelom hineinragend.

Weibliches Genitals ys tem : Ovarien kompakt, in normaler Position. Jeweils nur ein reifes Ei vorhanden. Spermatheken relativ einfach gebaut: Ampulle bei vollreifen Tieren rundlich-oval, Obergang zum Ausführgang gut abgegrenzt (Abb 1f). Bei subadulten Individuen und bei Körperstreckung Ampulle länglich gedehnt. Lumen in dieser Position sich allmählich zum Ausführgang hin verengend (Abb. 2c). Wandung der Apulle dick, Lumen weit. Der Ausführgang ist relativ kurz und bis zur Ausmündung deutlich erkennbar. Epithel des Ausführganges umgeben von einigen unterschiedlich großen Drüsenzellen, die zu etwa 10 bis 15 Stück am ectalen Teil besonders groß ausgebildet sind, ohne jedoch eine typische „Drüsenmanschette" $\mathrm{zu}$ bilden (Abb. 2c). Ampulle steht nicht mit Lumen des Oesophagus in Verbindung, sondern ist lediglich mit Gewebssträngen angeheftet. Spermien unregelmäßig im Zentrum des Ampullenlumens angeordnet.

Vorkommen und ökologische Bemerkungen: Der Fundort von Marionina istriae lag auf der Ostseite (Landseite) der Insel Kolone, ca. 5 sm südöstlich von Rovinj in Istrien. Die Würmer lebten in recht großer Zahl in den oberflächigen Schichten der supralitoralen Feuchtsandzone, ca. $50 \mathrm{~cm}$ oberhalb der Wasserlinie in einem sehr schill-reichen, recht groben Sand. Das semi-lotische Sediment besaß hohe Permeabilität und war gut durchfeuchtet mit voll marinem Wasser.

Der interstitiellen Lebensweise der Tiere entspricht ihre Fähigkeit, sich durch Drüsensekrete der Epidermis mit dem ganzen Körper an Sandkörner o. ä. anheften zu können. Bei Störungen rollen sich die Würmer zu einer Spirale auf (vgl. Marionina subterranea).

B e le g m a te ria 1: Die Artbeschreibung ist gegründet auf einer Typusserie von 13 reifen Individuen. Holotyp, fixiert und gefärbt nach ReIsinger (1925), hinterlegt in den Wissenschaftlichen Sammlungen des Zoologischen Instituts und Zoologischen Museums der Universität Hamburg, Katalog-Nr. Ol. 13 169. Die entsprechend behandelten 12 Paratypen tragen die Katalog-Nr. Ol. 13170.

\section{DISKUSSION}

\section{Systematische Stellung von Marionina istriae n. sp.}

Die oben beschriebene neue Art läßt sich durch folgende Merkmale zwanglos in das Genus Marionina einfügen: Gestalt und Anordnung der Borsten; Form der Nephridien mit großem Anteseptale und terminalem, kurzem Samenleiter; Anordnung der Septaldrüsen; Fehlen von Seminalvesikeln; Fehlen von Peptonephridien; relativ einfache Form der Spermatheka.

Für die Diskussion systematisch wichtiger Details werden im Genus Marionina neben Prüfung der Borstenarmatur, der Segmentzahl und Anordnung der Septaldrüsen üblicherweise die Ausbildung des Samentrichters, der Spermatheka und des terminalen 
Hirnumrisses als relevant angesehen und sollen daher hier nähere Erörterung finden: Die nach diesen Kriterien am ehesten für anatomische Vergleiche mit der neuen Art in Frage kommenden Marionina-Species werden im folgenden, der Anschaulichkeit halber in Tabellenform, gegen Marionina istriae abgegrenzt (Tab. 1) und in einigen Punkten näher erläutert.

Ein gerader $\mathrm{Hir}$ - $\mathrm{H}$ inter rand, für das Genus Marionina ein außergewöhnliches Merkmal (MichaELsEN 1905), ist bei Marionina sjaelandica ausgebildet (Tab. 1). Diese Art ist aber vor allem wegen völlig abweichender Form der Spermatheka und wegen anderer Segmentzahl nicht in nähere Beziehung zu M. istriae n. sp. zu bringen.

Für Marionina argentea gibt MICHAELSEN in seiner Originalbeschreibung (1889) ebenfalls einen geraden bis schwach konvexen Hinterrand an, betont aber nach Lebendbeobachtung dessen variable Form (vgl. p. 367). Die widersprüchlichen Angaben über den Hirnumriß von $M$. argentea bei Nielsen \& Christensen (1959) sind vielleicht aus der Variabilität dieses Merkmals zu erklären: Nach der Texterläuterung ist "the posterior end truncate or slightly convex", was mit $M$. istriae zu parallelisieren wäre. Die entsprechende Abbildung 141 allerdings zeigt deutlich einen tief ausgebuchteten Hirn-Hinterrand.

Ahnlichkeit in der Ausbildung des $\mathrm{S}$ a mentrichters läßt sich bei $M$. sjaelandica nachweisen, doch stimmen hier Form und Maße des Kragens nicht mit $M$. istriae überein, abgesehen von den deutlichen Seminalvesikeln.

Im systematisch wichtigsten Einzelmerkmal, der Ausformung der Spermathe ka, findet sich unter allen in Frage kommenden Arten keine Ubereinstimmung mit $M$. istriae. In der Ampullenform ist die neue Art zwar mit $M$. cana Marcus vergleichbar, doch betont Marcus (1965) die Abwesenheit jeglicher Drüsen am spermathekalen Ausführgang dieser südamerikanischen Art. Drüsenlose, in der Form aber ähnliche Spermatheken finden sich auch bei $M$. cliarensis (vgl. SoutHeRN 1913) und bei $M$. litterata (HESSE).

In geographischer Nachbarschaft von $M$. istriae lebt $M$. adriatica $=$ Enchytraeus adriaticus VejDovsky. Trotz Ahnlichkeit in der Form der Nephridien und Borsten (irrelevante Merkmale für die Species-Zuordnung), des Gehirns und der Gesamtlänge weist diese Art Divergenzen in der Borsten- und Segmentzahl sowie vor allem im Bau von Samentrichter und Spermatheka auf. Daher kommt eine Übereinstimmung mit der bei Rovinj gefundenen Art nicht in Betracht.

Bei der vor Neapel gefundenen $M$. litterata $=$ Parenchytraeus litteratus (Hesse 1893, vgl. auch C̈ennosvitov 1934), schließen Ausbildung der Spermatheka, des Samentrichters und des Gehirns eine Identität mit $M$. istriae aus.

Andere im Mittelmeergebiet gefundene marine Enchytraeiden [Enchytraeus mediterraneus MICHAELSEN; Lumbricillus litoreus (HESSE); Marionina achaeta (HAGEN)] stehen Marionina istriae zu fern, um näher in Betracht zu kommen. Die systematische Stellung der hier beschriebenen neuen Art innerhalb des Genus Marionina läßt sich folgendermaßen umreißen: Es bestehen offenbar verwandtschaftliche Beziehungen zwischen Marionina istriae und $M$. cana Marcus sowie M. litterata (Hesse). Ahnlichkeiten bestehen zweifellos auch zwischen $M$. cliarensis (SoutherN) und $M$. istriae (Gehirn, evt. auch Spermatheka und Nephridium), doch erlaubt die ungenügende Beschreibung der 
Ein mariner Enchytraeide aus dem Hygropsammal

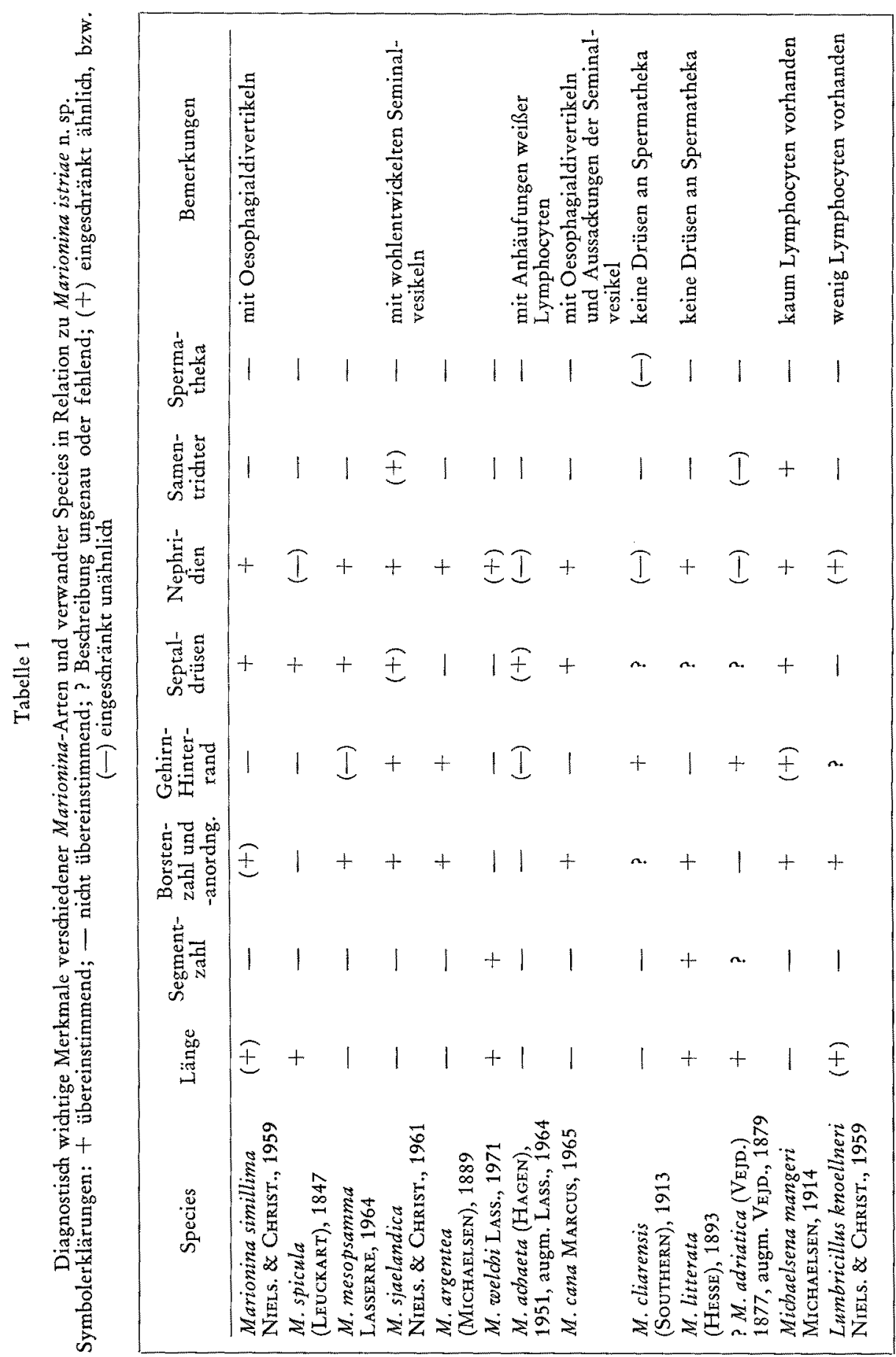




\begin{tabular}{|l|l|}
\hline Seminalvesikel & \\
Borsten - & \\
anordnung & \\
Septal - \\
drüsen \\
hinterrand
\end{tabular}

Abb. 3: Marionina istriae n. sp. Schematisches Diagramm diagnostisch wichtiger Merkmale 
irischen Art kein näheres Urteil (Borstenzahl?). Obwohl geographisch direkt benachbart, sind $M$. istriae und $M$. adriatica (VEjD.) deutlich gegeneinander abzugrenzen.

Die hier beschriebene Marionina istriae ist eine wohl abgegrenzte neue Enchytraeiden-Art des marinen Küstensaumes, speziell des Interstitials im Feuchtsand. Die Kombination der einzelnen diagnostisch relevanten Kriterien bringt die Eigenarten der neuen Species klar zum Ausdruck (Tab. 1).

Uberlegungen über die evtl. Verteilung oder gar Vorkommensgrenze der neuen Art können nicht angestellt werden, da das Material nur von einem Einzelfundort stammt. Generell erlaubt die fast völlig fehlende und dringend zu ergänzende Kenntnis über mediterrane Enchytraeiden keinerlei Beurteilung des Vorkommens mariner Enchytraeiden-Arten in dieser Region.

Eine Kurzdiagnose an Hand weniger determinatorisch entscheidender Schemazeichnungen, wie sie LASSERRE $(1966,1971)$ in so übersichtlicher und vorzüglicher Form entworfen hat, sei abschließend noch gegeben, um Marionina istriae in die Bestimmungs-Tabelle LASSERREs für marine interstitielle Marionina-Arten (1971) einfügen zu können (Abb. 3).

Bemerkungen zur Relevanz einiger anatomischer Merkmale für die Enchytraeiden-Taxonomie

Hirnum $\mathrm{r}$ i : Die Lebenduntersuchung an verschiedenen Exemplaren von Marionina istriae n. sp. zeigte, daß der Hirn-Hinterrand ein recht variables Merkmal ist. Seine "Normal-Konturierung" im Leben, die wahrscheinlich bei entspannter Lage der am Gehirn ansetzenden Muskelzüge zu beobachten ist, läßt sich nur aus der Summierung wiederholter Einzelbeobachtungen ableiten. In bestimmten Bewegungsphasen (Streckung bzw. Kontraktion) kann der Umriß durchaus verändert und somit auch gänzlich untypisch fixiert werden: Gelegentliche konvexe oder, seltener, konkave Form des Hinterrandes bei der vorliegenden Art (Abb. 1a und b). Daß die Darstellung solcher "momentanen Erscheinungsbilder" dieses Merkmals leicht in Artbeschreibungen hineingerät und zu irrtümlicher Wertung der Normalausformung führen kann, liegt auf der Hand.

Auf die Variabilität des Merkmals "Gehirn" wies schon Michaelsen (1889) bei Beschreibung von Marionina argentea und SOUTHERN (1913) bei Untersuchung von Enchytraeus cliarensis (= Marionina cliarensis, vgl. Cennosvitov 1934) hin. Eine taxonomische Relevanz des Merkmals "Hirnumriß" ist also nur bei sorgfältiger Lebenduntersuchung gegeben (vgl. NiELSEN \& CHRISTENSEN, 1959).

Verlauf der Blutgefäße im Vorderkörper: In der Originalbeschreibung von Parenchytraeus litteratus weist HESSE (1893) auf eigentümlich verzweigte Blutgefäße im Vorderkörper hin. Obwohl Nielsen \& Christensen (1959) diesem Merkmal keine besondere Bedeutung zumaßen, griff Marcus (1965) die Ausführungen Hesses auf und zog sie zur Species-Abgrenzung bei Marionina cana heran. Es erschien daher berechtigt, die bei MARcus kritiklos übernommenen Angaben von HessE einmal zu überprüfen: Hierbei zeigte sich, daß das „bei allen Enchytraeiden gleichmäßig vorhandene" Verzweigungsmuster (vgl. Abb. 11 bei HESSE) durchaus nicht generell gilt. 
Unter den im marinen Bereich häufigen Genera besitzen zwar auch Lumbricillus und Enchytraeus i. a. ein bis über das Gehirn hinweg ungeteilt verlaufendes Dorsalgefäß, das sich erst im ersten Segment aufteilt (s. Abb. 1d), doch gilt dieses Verzweigungsschema selten im Genus Marionina: So liegt bei Marionina istriae $\mathrm{n}$. sp. ebenso wie bei M. spicula, M. subterranea, $M$. achaeta die Aufzweigungsstelle des Dorsalgefäßes in die beiden vorderen Lateralschlingen etwa im 3. bis 4. Sement (vgl. Abb. 1c), was genau dem Gefäßsystem "von eigenartiger Beschaffenheit" (Fig. 1 in Hesse 1893) bei Marionina litterata (HEsse) und bei $M$. cana Marcus entspricht. Die ventrale Vereinigung der vorderen Lateralschlingen ist nahe der Grenze $4 / 5 \mathrm{zu}$ finden. Die nach Hesse und später MARcus angeblich ungewöhnliche Caudadverlagerung der dorsalen Gefäßaufzweigung ist also offenbar ein bei Marionina verbreitetes anatomisches Merkmal. Hierauf bezieht sich wahrscheinlich auch v. BüLow (1957), die sogar „eine besondere Anordnung des Blutgefäßsystems“ im ganzen Genus Michaelsena (= Marionina) als „klares Unterscheidungsmerkmal gegen Enchytraeus und Pachydrilus" (= Lumbricillus) wertet.

Es scheint allerdings auch unter den Marionina-Species einige zu geben, die im Blutgefäßverlauf dem für Lumbricillus und Enchytraeus typischen Bild entsprechen (Abb. 1d): z. B. Marionina cliarensis (Southern) und M. southerni (ČRnosvitov). Grundsätzliche Unterschiede zwischen den üblicherweise recht nahe verwandt gewerteten Genera Marionina und Lumbricillus scheinen in diesem Merkmal offenbar doch nicht zu bestehen. NIELSEN \& CHRISTENSEN (1959) schränken daher wohl mit Recht die taxonomische Relevanz des Blutgefäß-Bauplanes allgemein ein.

\section{ZUSAMMENFASSUNG}

1. Vom marinen Feuchtsand einer Insel nahe Rovinj (jugoslawische Adria) wird eine neue Enchytraeiden-Art, Marionina istriae n. sp. (Oligochaeta), beschrieben. Die Abgrenzung als eigene Art wird begründet und ihre Stellung innerhalb des Systems diskutiert.

2. Eine Kurzdiagnose an Hand einiger Schema-Zeichnungen soll die neue Art in die Zusammenstellung der interstitiellen Marionina-Arten von LASSERRE (1971) einfügen.

3. Die Relevanz der diagnostischen Kriterien „Hirnumriß“ und „Blutgefäß-Aufzweigung" wird geprüft.

Danksagung. Für die Uberlassung des Materials und für interessante Hinweise danke ich Herrn cand. rer. nat. U. Graefe, Hamburg.

\section{ZITIERTE LITERATUR}

BüLow, TH. von, 1957. Systematisch-autökologische Studien an eulitoralen Oligochaeten der Kimbrischen Halbinsel. Kieler Meeresforsch. 13, 69-116.

Černosvirov, L., 1934. Zur Kenntnis der Enchytraeiden II. Zool. Anz. 105, 295-305.

Hesse, R., 1893. Beiträge zur Kenntnis des Baues der Enchytraeiden. Z. wiss. Zool. 57, 1-17. 
LASSERkE, P., 1964. Note sur quelques Oligochètes Enchytraeidae, présents dans les plages du bassin d'Arcachon. P.-V. Soc. linn. Bordeaux 101, 1-5.

- 1966. Oligochètes marins des côtes de France. I. Bassin d'Arcachon: Systématique, Cah. Biol. mar. 7, 295-317.

- 1971. Oligochaeta from the marine meiobenthos: Taxonomy and ecology. Smithson. Contr. Zool. 76, 71-86.

Marcus, E., 1965. Naidomorpha aus brasilianischem Brackwasser. Beitr. neotrop. Fauna 4, 61-83.

Mrchaelsen, W., 1889. Oligochaeten des Naturhistorischen Museums in Hamburg. I. Jb. hamb. wiss. Anst. 6, 1-17.

- 1905. Die Oligochaeten der schwedischen Südpolar-Expedition. Wiss. Ergebn. schwed. Südpolarexped. 5 (3), 1-12.

- 1914. Beiträge zur Kenntnis der Land- und Süßwasserfauna Deutsch-Südwestafrikas. Oligochaeta. Ergebn. Hamb. Deutsch-Südwestafrik. Studienreise. Friedrichsen, Hamburg, 1911, 139-182.

- 1925. Zur Kenntnis einheimischer und ausländischer Oligochaeten. Zool. Jb. (System. Okol. Geogr. Tiere) 51, 255-328.

Nizlsen, C. O. \& Christensen, B., 1959. The Enchytraeidae. Critical revision and taxonomy of European species (Studies on Enchytraeidae VII). Natura jutl. 8-9, 1-160.

- 1961. The Enchytraeidae. Critical revision and taxonomy of European species. (Suppl. 1). Natura jutl. 10, 1-23.

ReIsinger, E,, 1925. Ein landbewohnender Archiannelide. (Zugleich ein Beitrag zur Systematik der Archianneliden.) Z. Morph. Okol. Tiere 3, 197-254.

Southern, R., 1913. Clare Island survey, Pt. 48. Oligochacta. Proc. R. Irish Acad. 31, 1-14. VejDovsKY, F., 1877. Zur Anatomie und Systematik der Enchytraeiden. Sber. K. böhm. Ges. Wiss. 1877, 294-304.

- 1879. Beiträge zur vergleichenden Morphologie der Anneliden. I. Monographie der Enchy* traeiden. Tempsky, Prag, $61 \mathrm{pp}$.

Anschrift des Autors: Dr. O. Giere

Zoologisches Institut und Zoologisches Museum

Universität Hamburg

2 Hamburg 13

Papendamm 3

Bundesrepublik Deutschland 